intestinal) and time since non-Raynaud phenomenon. Clinical characteristics and prevalence of auto-antibodies within clusters was evaluated. We assessed whether adding autoantibody status as additional factor improved clinical subsetting. Results: Of 407 SSc patients included, 371 patients (91\%) fulfilled ACR/EULAR 2013 criteria. Prevalence of anti-centromere (ACA) was $37 \%$, anti-topoisomerase (ATA) $24 \%$, anti-RNAPIII $5 \%$, anti-fibrillarin $4 \%$, anti-Pm/Scl $5 \%$. Cluster analysis identified clinically recognisable clusters of SSc patients based on absence of skin involvement (cluster 1), peripheral vascular involvement (cluster 2), fibrotic complications (cluster 4), and severe vascular complications (pulmonary arterial hypertension [PH], renal crises; cluster 5). Except for cluster 4, where ATA was dominant, in all clusters ACA was the most prevalent auto-antibody. No cluster associated with any of the more rare SSc specific auto-antibodies. Adding of auto-antibodies resulted in increased clinical overlap, with a frequency of $\mathrm{PH}$ between $6-8 \%$ and a frequency of digital ulcers between $24-39 \%$ in clusters 2,4 and 5 .

Table 1. Clustering of SSc patients based on clinics

\begin{tabular}{lccccc}
\hline & $\begin{array}{c}\text { Cluster 1 } \\
(\mathrm{n}=66)\end{array}$ & $\begin{array}{c}\text { Cluster 2 } \\
(\mathrm{n}=50)\end{array}$ & $\begin{array}{c}\text { Cluster 3 } \\
(\mathrm{n}=129)\end{array}$ & $\begin{array}{c}\text { Cluster } 4 \\
(\mathrm{n}=81)\end{array}$ & $\begin{array}{c}\text { Cluster 5 } \\
(\mathrm{n}=81)\end{array}$ \\
\hline Age in years, mean (SD) & $53(14)$ & $54(14)$ & $54(14)$ & $53(16)$ & $62(12.4)$ \\
Female, \% (n) & $90(60)$ & $86(43)$ & $85(110)$ & $72(58)$ & $74(60)$ \\
Time since non-Raynaud, median (range) & $3(-3-14)$ & $10(-3-44)$ & $2(0-20)$ & $3(0-27)$ & $13(0-46)$ \\
dcSSc, \% (n) & $0(0)$ & $2(1)$ & $17(22)$ & $65(53)$ & $25(20)$ \\
Mean mRSS (SD) & $0(0)$ & $4(3)$ & $4(3)$ & $14(11)$ & $4(6)$ \\
PAH, \% (n) & $3(2)$ & $4(2)$ & $0(0)$ & $5(4)$ & $20(16)$ \\
DLCO, \%predicted, mean (SD) & $70(18)$ & $66(16)$ & $70(15)$ & $64(16)$ & $54(18)$ \\
History of renal crisis, \% (n) & $0(0)$ & $0(0)$ & $0(0)$ & $3(2)$ & $17(14)$ \\
Digital ulcers, \% (n) & $15(10)$ & $98(49)$ & $4(5)$ & $28(23)$ & $6(5)$ \\
Anti-topisomerase, \% (n) & $5(3)$ & $15(7)$ & $22(28)$ & $48(37)$ & $24(19)$ \\
Anti-centromere, \% (n) & $62(39)$ & $48(23)$ & $41(53)$ & $8(6)$ & $30(24)$ \\
\hline
\end{tabular}

Conclusions: Based on clinical data alone, relevant subgroups in SSc can be distinguished. Adding antibody status to cluster analyses increases overlap of clinical features between subgroups. These findings show that solely presence of SSc specific antibodies is of limited clinical relevance. For optimal risk stratification more complex serological findings as antibody titers might be additive.

Disclosure of Interest: None declared

DOI: 10.1136/annrheumdis-2017-eular.6004

\section{SAT0351 DIFFERENCES AMONG PATIENTS WITH INTERSTITIAL LUNG DISEASE ACCORDING TO THEIR SYSTEMIC SCLEROSIS SUBCLASSIFICATION}

M. Laffont ${ }^{1}$, I. Catellvi ${ }^{2}$, D. Castillo ${ }^{3}$, M. Millan ${ }^{2}$, C. Diaz-Torné ${ }^{2}$, P. Moya ${ }^{2}$, B.P. Magallares ${ }^{2}$, A. Laiz ${ }^{2}$, H.S. Park ${ }^{2}$, J.M. De Llobet $^{2}$, J. Casademont ${ }^{2}$ ${ }^{1}$ Reumatologia, Hospital Jose Maria Ramos Mejia, Ciudad Autónoma de Buenos Aires, Argentina; ${ }^{2}$ Reumatologia; ${ }^{3}$ Neumonologia, Hospital Universitario de la Santa Creu I Sant Pau, Barcelona, Spain

Background: Systemic Sclerosis (SSc) has been widely studied from a purely global standpoint, but only a few trials have analysed patients with interstitial lung disease (SSc-ILD) as well.

Objectives: 1. Describe the clinical features of a cohort of patients with SSC and SSc-ILD. 2. Determine whether there are disparities among different types of SSC-ILD and their progression in patients with either limited (ICSSc) or diffuse involvement (dcSSc). 3. Ascertain whether there are disparities among different types of SSc-ILD and their progression according to the SSc-specific autoantibody (AAb).

Methods: Retrospective study of a cohort of patients with ILD-SSc controlled during an SSc consultation. The following variables were collected: sex, age, SSc and ILD progress in years, type of SSc and ILD, smoking, digital ulcers (DU), pulmonary hypertension, digestive disorders, cancer, SSc treatment, corticosteroid doses and lastly, lung function tests upon diagnosis, at treatment onset, and 24 months later. Additionally, a record was kept on the types of AAb present in every SSc. Qualitative and quantitative variables were compared in relation to the clinical and immunological sub-classification. Chi-square and Student's T Tests were performed. A p-value $\leq 0.05$ was considered significant.

Results: out of 266 patients with SSc, data from 47 patients with ILD-SSc were gathered; $89.4 \%$ were female, with an age range of $66.09 \pm 15.1$ years old, and $9.85 \pm 10.2$ and $4.38 \pm 9.24$ years of progression of their SSc and ILD respectively. 33 out of 47 sustained IcSSc, and both Scl-70/ATA (29.8\%) and ACA (26.1\%) were the most frequently found AAb. Non-specific interstitial pneumonia (NSIP) was the most common ILD radiological pattern (76.6\%). Most patients with SSc underwent treatment $(51.1 \%), 24 \%$ with mycophenolate mofetil (MMF); $36.2 \%$ of the patients had been concomitantly administrated corticosteroids with a mean prednisone dose of $15.73 \pm 10.3 \mathrm{mg} / \mathrm{d}$.

Upon comparing patients with IcSSc and dcSSc, prevalence of DU was higher in those with dcSSc $(p<0.01)$, MMF was less frequently used $(p<0.02)$, rituximab was more usually employed $(p<0.03)$, and they presented worse values of FEV1/FVC ratio after 24 months of treatment $(p<0.03)$. No differences were observed as to either type of ILD or progression. However, when variables were analysed regarding AAb in SSc, patients with ACA presented both fewer DU $(p<0.02)$ and NSIP pattern $(p<0.02)$, and more frequent compromise of the small airway $(p<0.01)$, they were younger and thus, they had had shorter progression of the disease. ILD diagnosis was made significantly earlier in those patients with RNA polymerase, and later in those with anti-U1RNP. No AAb was observed associated with neoplasia. Considering the types of ILD, patients with NSIP pattern were younger ( $\mathrm{p} 0.054)$ and presented worse spirometric values.

\begin{tabular}{|c|c|c|c|c|c|c|c|c|}
\hline & $\begin{array}{l}170-5 s c \\
n-47\end{array}$ & 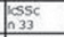 & $\begin{array}{l}\text { bessc } \\
14 \\
14\end{array}$ & 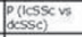 & $\begin{array}{l}\text { P(ACA vs no } \\
\text { ACA) }\end{array}$ & $\begin{array}{l}\text { P(ATA van no } \\
\text { ATA) }\end{array}$ & 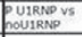 & 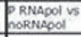 \\
\hline Age \pm So (years) & $\begin{array}{l}\begin{array}{l}66.09 \pm \\
14.1\end{array} \\
\end{array}$ & $\begin{array}{l}67.09 \pm \\
14.04\end{array}$ & $63.71 \pm 15.67$ & Ns & $<0.05$ & Nos & Ws & is \\
\hline rogress in years $\mathrm{SSc} \pm \mathrm{SO}$ & $\begin{array}{l}9.85 t \\
10.29\end{array}$ & $\begin{array}{l}3.81 \pm 15 \\
04\end{array}$ & $12.31=9.59$ & Ns & $<0.04$ & Ns & $<0.02$ & is \\
\hline Evodution in years $\mathrm{HD} \pm \mathrm{SD}$ & $\begin{array}{l}4.38 \pm \\
9.24\end{array}$ & $\begin{array}{l}4.11 \pm 9.7 \\
2\end{array}$ & $\begin{array}{l}5.02 \pm \\
3.29\end{array}$ & Ns & Ns & is & $<0.0001$ & $<0.005$ \\
\hline $\operatorname{NST}(\%)$ & $36(76.6)$ & $(72.72)$ & $12(85.71)$ & Nos & $<0.02$ & is & is & is \\
\hline Ff (\%) & $3(6,4)$ & $1(3.03)$ & $2(14.28)$ & Ns & Ns & Wis & Wis & is \\
\hline$\Phi P(\%)$ & $2(4,3)$ & $2(6.06)$ & 6 & Ns & Nis & Nis & is & $4 s$ \\
\hline Small arway compromise(\%) & $4(8.5)$ & $4(12.12)$ & p & ws & $<0.005$ & Ns & is & is \\
\hline PH (ultraound) & $3(6.4)$ & $3(9.09)$ & to & Ns & Ns & Nos & ws & ws \\
\hline pigitd ulcors (5) & $\begin{array}{l}17 \\
(36.2) \\
\end{array}$ & $7(21.21)$ & $10(71,42)$ & $<0.01$ & $<0.02$ & $<0.01$ & Wis & Wis \\
\hline Neoplasa (\%) & $5(10.6)$ & $3(9.09)$ & $2(14.28)$ & Ns & Ns & Ns & Hs & is \\
\hline $\begin{array}{l}\text { mminosuppressive } \\
\text { treament(5) }\end{array}$ & $\begin{array}{l}24 \\
(51.1)\end{array}$ & $\begin{array}{l}18 \\
(54.54) \\
\end{array}$ & $0(42.85)$ & Nos & Nos & wis & His & is \\
\hline Use of cylophosphamide (\%) & $8(17)$ & $4(12.12)$ & $4(28.57)$ & Ns & Ns & Nos & ws & is \\
\hline Use of MMF (\%) & $16(34)$ & $\begin{array}{l}125 \\
(45.45) \\
\end{array}$ & $1(7.14)$ & $<0.02$ & Ns & ws & us & vs \\
\hline Use of azathiogrine (\%) & $1(24)$ & 0 & $1(7.14)$ & Nis & Ns & Ns & us & is \\
\hline Use of riturim $3 b$ (s6) & $3(6.4)$ & 0 & $3(21.42)$ & $<0.03$ & Ns & $<0.03$ & is & is \\
\hline Use of corbooids (56) & $17(36.2)$ & $\begin{array}{l}13 \\
(39.39) \\
\end{array}$ & $4(28.57)$ & Ns & Ns & Ns & is & is \\
\hline $\begin{array}{l}\text { Doses of corbcoods } \pm \text { SD } \\
\text { molos }\end{array}$ & \begin{tabular}{|l}
$15.73 \mathrm{z}$ \\
10.31
\end{tabular} & $\begin{array}{l}14.42 \mathrm{t} \\
0.79\end{array}$ & $\begin{array}{l}200 \\
12.24\end{array}$ & Ns & $<0.02$ & Nis & Us & is \\
\hline $\begin{array}{l}\text { SFVC } \pm S D \text { upon diagnois of } \\
S S C\end{array}$ & $\begin{array}{l}32.29 \pm \\
14.6\end{array}$ & $\begin{array}{l}34.54 \\
13.41 \\
\end{array}$ & $77.89 \pm 16.67$ & Nis & Ns & Nis & Eis & $<0.001$ \\
\hline $\begin{array}{l}\text { WFivctso upon diagnosis of } \\
\text { iD }\end{array}$ & $\begin{array}{l}77.8 \pm \\
16.09\end{array}$ & $\begin{array}{l}80.292 \\
18.13 \\
\end{array}$ & $1.92 \pm 13.96$ & Nos & Nos & Nis & Wis & is \\
\hline $\begin{array}{l}\text { WFVCASD } 24 \text { months afort } \\
\text { diannosis of } 10\end{array}$ & $\begin{array}{l}77.65 \pm \\
20.94 \\
\end{array}$ & $\begin{array}{l}79.85 \pm \\
22.6\end{array}$ & $73.45 \pm 17.58$ & ws & Ns & Nis & us & vs \\
\hline $\begin{array}{l}\text { WFEVI/FVCE SO UPOn } \\
\text { diagnosis of SSC }\end{array}$ & $\begin{array}{l}83.78 t \\
14.07 \\
\end{array}$ & $\begin{array}{l}85.111 \\
15.71 \\
5.12\end{array}$ & $31.11 \pm 10.21$ & $N$ & $N=$ & Ns & is & $y=$ \\
\hline $\begin{array}{l}\text { EFFEVI/FVCTSO UDON } \\
\text { Jiagnosis of ILD }\end{array}$ & $\begin{array}{l}37.79 \pm \\
16.06\end{array}$ & $\begin{array}{l}89.565 \\
10.85 \\
\end{array}$ & $33.69 \pm 13.79$ & Ns & Ns & Nis & sis & is \\
\hline $\begin{array}{l}\text { STFEVI/FVCA SD } 24 \text { manths } \\
\text { ffter diagnosis of ILO }\end{array}$ & $\begin{array}{l}93.25 \pm 1 \\
8.93\end{array}$ & $\begin{array}{l}98.71 \pm 1 \\
7.24\end{array}$ & $\begin{array}{l}31.8 \mathrm{~s} \\
17.83 \\
\end{array}$ & $<0.03$ & Ns & 0.08 & is & is \\
\hline $\begin{array}{l}\text { WDLCOA SD upon Giagnosis } \\
\text { of SSC }\end{array}$ & \begin{tabular}{|l}
$\begin{array}{l}59.76 t \\
17.8\end{array}$ \\
\end{tabular} & $\begin{array}{l}70.93 \pm \\
18.46\end{array}$ & $67.67 \pm 17.42$ & Ns & Ns & Ns & ys & is \\
\hline $\begin{array}{l}\text { MoLCO SO upon diagnosis } \\
\text { fItLO }\end{array}$ & $\begin{array}{l}63.28 \mathrm{t} \\
18.31 \\
\end{array}$ & \begin{tabular}{|l}
$65.69 *$ \\
18.75
\end{tabular} & $57.92 \pm 16.75$ & Ns & Ns & Nis & is & ws \\
\hline 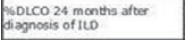 & $\mid \begin{array}{l}61.31 * \\
17.47\end{array}$ & $\begin{array}{l}\text { 63.t } \\
18.74\end{array}$ & $58.45 \pm 15.68$ & Ns & Ns & Nis & bs & is \\
\hline
\end{tabular}

Conclusions: In terms of ILD-SSc patient stratification, sub-classification by AAb appears to be more specific than the clinical sub-classification. ACA is related to less frequency of NSIP pattern. Unlike what has been described for SSc from a global point of view, in patients with ILD-SSc no association between AAb and neoplasia could be established.

Disclosure of Interest: None declared

DOI: 10.1136/annrheumdis-2017-eular.6138

\section{SAT0352 MORTALITY IN IDIOPATHIC INFLAMMATORY MYOPATHY- RESULTS FROM A SWEDISH NATIONWIDE POPULATION-BASED COHORT STUDY}

C. Dobloug ${ }^{1}$, J. Svensson ${ }^{2}$, I. Lundberg ${ }^{2}$, M. Holmqvist ${ }^{2}$. ${ }^{1}$ Oslo University Hospital, Oslo, Norway; ${ }^{2}$ Karolinska Institutet, Stockholm, Sweden

Background: Little is known about mortality in idiopathic inflammatory myopathies (IIM) compared to the general population, especially the risk development since diagnosis. In a recently published study, the 5-, and 10-year mortality was lower for IIM than previously reported, suggesting improved outcomes in recent years. Objectives: To assess the mortality following IIM diagnosis in a nationwide population-based cohort of IIM patients diagnosed 2002-2011 compared to the general population.

Methods: We used nationwide fully covering health care registers to identify all individuals who were treated by a rheumatologist, neurologist, or dermatologist for IIM and who had a listing of IIM at $\geq$ two visits within 1-12 months between 2002 and 2011, general population comparators, and death during follow up. We calculated mortality rates with $95 \%$ confidence intervals $(\mathrm{Cl})$ and compared the mortality in IIM to that in the general population using Cox proportional hazards models adjusted for age, sex, year of diagnosis, and residential area.

Results: During a median follow up of 4 years, $228(31 \%)$ of the 733 IIM patients and during a median follow up of 6 years, $888(12 \%)$ of the 7,340 general population comparators died. This corresponded to a crude mortality of $61 / 1,000$ person-years in IIM, and 20/1,000 person-years in the comparators. The cumulative mortality at 1 year after diagnosis was $10 \%$ in IIM and $1 \%$ in the general population, at 5 years it was $24 \%$ in IIM and $7 \%$ in general population, and at 10 years the proportion was $31 \%$ and $12 \%$. The overall hazard ratio (HR) $(95 \% \mathrm{Cl})$ of death comparing the NPR cohort and its comparator, was 3.5 (3.0-4.0). When restricting the outcome to cardiovascular disease-, cancer-, infection-, or pulmonary disease- specific death we noted increased mortality from all outcomes in IIM compared to the risk in the general population. When we stratified on time since diagnosis we noted an increased absolute and relative risk of death in the first year of diagnosis in particular for pulmonary disease and cancer, whereas cardiovascular mortality was a major cause of death also after 10 years after IIM diagnosis (table).

Conclusions: IIM patients are still at increased risk of death. The highest mortality 
Abstract SAT0352 - Table 1. Hazard ratio (HR) and 95\% confidence intervals (CI) comparing the mortality in idiopathic inflammatory myopathy (IIM) patients identified in the National Patient register between 2002 and 2011 and in an individually matched general population comparator. Overall mortality and stratified by underlying cause of death and time since IIM diagnosis

\section{IIM duration categories}

$\mathrm{HR}(95 \% \mathrm{Cl})$

Deaths per 1,000 person years IIM patients/comparators

\begin{tabular}{cccc}
\hline$<1$ year & $1-<5$ years & $5-10$ years & $>10$ years \\
$10.3(7.5-14.2) 110.8 / 11.6$ & $3.2(2.5-4.0) 51.2 / 19.6$ & $2.6(1.8-3.6) 48.2 / 24.7$ & $2.4(0.6-9.2) 42.1 / 27.7$ \\
$5.3(2.8-9.9) 21.9 / 4.4$ & $2.3(1.6-3.5) 14.9 / 8.0$ & $2.4(1.4-4.2) 16.7 / 10.0$ & $6.49(1.1-40.0) 28.1 / 13.4$ \\
$12.4(7.4-20.8) 46.7 / 4.2$ & $3.2(2.1-4.8) 15.4 / 5.5$ & $3.1(1.6-6.0) 12.8 / 5.6$ & $1.5(0.1-14.9) 14.0 / 6.7$ \\
$22.49(4.2-119.7) 7.3 / 0.1$ & $5.2(2.5-10.8) 6.1 / 0.3$ & $2.4(0.7-8.5) 3.0 / 1.0$ &.-
\end{tabular}

was noted within a year of diagnosis due to pulmonary disease, cancer and cardiovascular disease which calls for extra vigilance with respect to comorbidities during the first year of IIM diagnosis.

Disclosure of Interest: None declared

DOI: 10.1136/annrheumdis-2017-eular.2581

\section{SAT0353 SMALL AIRWAYS INVOLVEMENT IN SCLERODERMA} PATIENTS: RESULTS OF A CASE-CONTROL STUDY

M. Bonifazi ${ }^{1}$, M. Mattioli ${ }^{2}$, P. Fraticelli ${ }^{3}$, C. Fischetti ${ }^{2}$, L. Paolini ${ }^{1}$, S. Gasparini ${ }^{1}$, A. Gabrielli ${ }^{2}$. 'Pulmonary Diseases Unit, Department of Internal Medicine, Azienda Ospedaliero-Universitaria "Ospedali Riuniti"; ${ }^{2}$ Department of Biomedical Sciences and Public Health, Università Politecnica delle Marche, Ancona: ${ }^{3}$ Clinica Medica, Department of Internal Medicine, Azienda Ospedaliero-Universitaria "Ospedali Riuniti", Ancona, Italy

Background: Interstitial lung disease (ILD) and pulmonary hypertension are the leading cause of morbidity and mortality in systemic sclerosis (SSc). Although a ventilatory obstructive pattern, due to large airways impairment, has been rarely observed in SSc, a potential involvement of smaller airways (SA) has been suggested in previous reports. Recently, impulse oscillometry (IOS), a non-invasive forced oscillation technique, has been advocated as a valuable diagnostic tool for a sensitive assessment of SA.

Objectives: The main objectives of the present study was to investigate the prevalence of SA dysfunction by IOS in SSc patients compared to healthy controls, and to evaluate the correlation between SA dysfunction and selected radiological and clinical disease-related features.

Methods: Consecutive SSc patients were included in the present study according to eligibility criteria; controls were health volunteers. Both cases and controls underwent IOS measurements; cases also underwent pulmonary function tests and St. George's respiratory questionnaire. Radiological features were assessed on the latest chest high resolution computed tomography (HRTC) scan available in the twelve months before study enrolment, evaluating for both SA signs of disease and ILD. A SA involvement at IOS was defined as R5-R20 $\geq 0.07 \mathrm{kPa} / \mathrm{L} / \mathrm{sec}$. Odds ratios and $95 \%$ confidence intervals for the IOS value was computed using multiple logistic regression models. Correlation between SA dysfunction and selected parameters were assessed using Pearson's correlation coefficient.

Results: 92 cases (M/F 14/78, mean age 57.06) and 84 controls (M/F 15/69, mean age 54.28) were included in the present study. The R5-R20 $\geq 0.07 \mathrm{kpa} / \mathrm{L} / \mathrm{sec}$ was found in $20.65 \%$ of cases and in $3.57 \%$ of controls. The OR was 7.027 $(95 \% \mathrm{Cl} 1.99-24.72, \mathrm{p}<0.01)$. This value did not significantly change after the adjustment for confounding variables (OR $a^{*} 7.091$ ). Correlations between R5-20 $\geq 0,07 \mathrm{kPa} / \mathrm{L} / \mathrm{s}$ and selected parameters showed a significant inverse association with vital capacity (FVC) and forced expiratory volume in first second (FEV1) and a direct correlation with pulmonary artery systolic pressure estimated. With reference to cutaneous subtype a SA dysfunction was more prevalent in the limited form compared to the diffuse, respectively in $23 \%$ and $12 \%$. Radiologic HRCT assessment of SA pathological features and ILD extent were provided for 77 patients: 19 (24.7\%) presented at least one sign of SA disease. An underlying ILD was detected in 40 patients, characterized by NSIP pattern in 37.

Conclusions: A significant involvement of SA was found in a substantial proportion of SSc patients, compared to healthy controls. Moreover, this seemed to be associated with a more severe functional obstructive and restrictive impairment, and with higher PAPs values. Therefore, our findings suggests that SA may be a potential, less known, target of disease, and further studies are needed to assess prognostic and therapeutic implications of this pathologic feature.

Disclosure of Interest: None declared

DOI: 10.1136/annrheumdis-2017-eular.5385

\section{SAT0354 NAILFOLD VIDEOCAPILLAROSCOPY AND RAYNAUD'S PHENOMENON IN A COHORT OF MESTIZO LATIN AMERICAN PATIENTS: A PRELIMINARY OBSERVATIONAL STUDY}

C.J. Velásquez-Franco ${ }^{1}$, A. Facio-Lince García ${ }^{2}$, A.L. Zapata-Castellanos ${ }^{1}$, L.M. Rodríguez-Padilla ${ }^{2}$, M.A. Mesa-Navas ${ }^{1}$ on behalf of Clinical Immunology and Rheumatology Group. Universidad Pontificia Bolivariana. ${ }^{1}$ Rheumatology, Clinica Universitaria Bolivariana. Universidad Pontificia Bolivariana; ${ }^{2}$ School of Health Sciences. Universidad Pontificia Bolivariana, Medellin, Colombia

Background: The prevalence of Raynaud's phenomenon (RP) has been reported between $3-22 \%$. When associated with systemic autoimmune diseases (SAD), especially systemic sclerosis (SSc), it is the sentinel event of irreversible organic damage. Nailfold videocapillaroscopy (NVC) is a non-invasive and safe procedure that allows in vivo observation of the microcirculation. Between $15-20 \%$ of patients who have RP with videocapillaroscopic alterations and certain autoantibodies will develop a SAD over two years. In addition, $90 \%$ of individuals with SSc and $85 \%$ with mixed connective tissue disease (MCTD) had RP as the first symptom.

Objectives: To evaluate the role of NVC in the differential diagnosis of RP, as well as in the early detection of SAD, in a cohort of Colombian patients.

Methods: A prospective, longitudinal, analytical study was conducted in subjects with RP, over 18-year-old, not active smokers, without previous connective tissue disease, secondary causes or aggravating factors. Optilia NVC with OptiPix software was used (Optilia Instruments; Sollentuna, Sweden). Qualitative variables are described by means, as well as absolute and relative frequencies; quantitative variables, according to the distribution of data, were reported by means or median, with standard deviation (SD) and interquartile range (IQR), respectively. We are reporting the baseline characteristics of these individuals. Results: Fifty-eight individuals were included; $91.4 \%$ were female. The mean age was 40.9 years (SD: 14.1 ). RP was biphasic in $63.6 \%$ of the patients, with a median of 30 episodes per month (IQR: 8-30). In 41 subjects (available data), antinuclear antibodies were positive; the most common patterns were: speckled $(41.5 \%)$ and centromere $(26.8 \%)$. The median of erythrosedimentation rate (ESR) was 9 (IQR 4-13). Ten individuals (19.2\%) were diagnosed with SAD in the first NVC: Seven patients with limited SSc, two with MCTD, and one with diffuse SSc. The patterns observed in the individuals with SSc were: early $(n=3)$, active $(n=3)$, late $(n=2)$, and minor and unspecific abnormalities in subjects with MCTD (one each). The most frequent NCV alterations in subjects with SAD were: megacapillaries $(n=10)$, microhemorrhages $(n=10)$, avascular zones $(n=8)$, neovascularization $(n=6)$, and capilar disorganization $(n=6)$. In these subjects, the mean capillary diameter was $76.7 \pm 33.9 \mathrm{~mm}$; the median of capillary number per mm was 7 (IQR: 6-8).

Conclusions: The frequency of systemic autoimmune disease was similar to the published reports in the literature. We highlight the following aspects: 1) The normal erythrosedimentation rate in subjects with a rheumatologic diagnosis, a particular finding when compared to previous data; 2) The important percentage of subjects with a specific diagnosis in the first nailfold capillaroscopy; one possible explanation could be a underdiagnosed disorder; this fact could be possibly demonstrated by the large capillary diameter found.

\section{References:}

[1] Ingegnoli F et al. Arthritis Rheum. 2008; 58 (7): 2174-82.

[2] Ingegnoli $F$ et al. Rheumatology 2010; 49 (4): 797-805.

Acknowledgements: Clinica Universitaria Bolivariana. Universidad Pontificia Bolivariana.

Disclosure of Interest: None declared

DOI: 10.1136/annrheumdis-2017-eular.4075

\section{SAT0355 NEUROPATHIC PAIN: IS IT AN UNDERESTIMATED SYMPTOM IN SYSTEMIC SCLEROSIS?}

N. Cuzdan Coskun ${ }^{1}$, I. Turk $^{2}$, T. Sarpel ${ }^{1}$, E. Erken ${ }^{2}$, Z.N. Alparslan ${ }^{3}$.

${ }^{1}$ Department of Physical Therapy and Rehabilitation, Division of Rheumatology; ${ }^{2}$ Department of Internal Medicine, Division of Rheumatology; ${ }^{3}$ Department of Biostatistics, Cukurova University Faculty of Medicine, Adana, Turkey

Background: Pain is one of the most common symptoms in SSc patients, yet not considered in the assessment of disease severity. Former studies have shown that pain has a neuropathic component; however there is still lack of evidence about its distribution in the body regions and the direct effect of neuropathic pain on the quality of life (QoL).

Objectives: We aimed to investigate the frequency of neuropathic pain syndrome (NPS) and to evaluate its interference with the quality of life in SSc patients.

Methods: Diffuse and Limited SSc patients diagnosed by American College of Rheumatology 2013 criteria were included in the study. Pain was evaluated with Visual Analogue Scale (VAS); painful body regions and pain intensity with Brief Pain Inventory (BPI); presence of neuropathic pain with The Leeds Assessment of Neuropathic Symptoms and Signs (LANNS) questionnaire; disease activity with Medsger Disease Severity Scale and QoL with Short-form 36 (SF36). Multiple regression analysis was used to assess the associations of NPS with sociodemographic and clinical factors.

Results: One hundred twenty patients were included in the study (mean age $53.64 \pm 11.44$ years, female/male $83.3 \%>16.7 \%$ ). Total pain frequency was found $69.2 \%$ and NPS was $35.9 \%$ in the whole patient group. Mean VAS in the 